\title{
CS Resarach Square \\ Predicting Hospital Mortality and Length of Stay: The Delirium Screening Checklist versus Confusion Assessment Method for Intensive Care Units
}

Hsiu Ching Li

National Taiwan University

Cheryl Chia-Hui Chen

National Taiwan University

Tony Yu-Chang Yeh

National Taiwan University Hospital

Shih-Cheng Liao

National Taiwan University Hospital

Adrian-Shengchun Hsu

National Taiwan University Hospital

Yu-Chung Wei

National Changhua University of Education

Shiow-Ching Shun

National Taiwan University

Shih-Chi Ku (D Scku1015@gmail.com)

https://orcid.org/0000-0002-4123-0967

Sharon K Inouye

Beth Israel Deaconess Medical Center

\section{Research}

Keywords: ICDSC (Intensive Care Delirium Screening Checklist), CAM-ICU (Confusion Assessment Method for Intensive Care Unit), Mortality, Delirium, Consciousness, RASS (Richmond Agitation-Sedation Scale)

Posted Date: August 2nd, 2021

DOl: https://doi.org/10.21203/rs.3.rs-754291/v1

License: (c) (i) This work is licensed under a Creative Commons Attribution 4.0 International License.

Read Full License 


\section{Abstract}

Background: Both the intensive care delirium screening checklist (ICDSC) and confusion assessment method for ICU (CAM-ICU) are valid tools for identification of delirium, however their relative predictive validity for important delirium outcomes, such as hospital mortality and LOS have not been wellestablished. We aim to compare the two tools for their predictive validity for outcomes related to delirium, hospital mortality and length of stay (LOS).

Methods: The prospective cohort study conducted in six medical ICUs at a tertiary care hospital in Taiwan. The study enrolled consecutive adult patients ( $\geq 20$ years) who were delirium free at ICU admission. Delirium was screened daily by trained research nurses using the ICDSC and CAM-ICU in random order. Arousal was assessed by the Richmond Agitation-Sedation Scale (RASS). Participants with any one positive result were classified as ICDSC- or CAM-ICU-delirium groups, respectively.

Results: Delirium incidence evaluated by the ICDSC and CAM-ICU were 69.1\% (67/97) and 50.5\% (49/97), respectively. Although the ICDSC identified 18 more cases as delirious, substantial concordance $(\mathrm{K}=0.63$; $\mathrm{p}<0.001$ ) was found between tools. Independent of age, APACHE II score, and Charlson comorbidity index, both ICDSC- and CAM-ICU-rated delirium significantly predicted hospital mortality (adjusted odds ratio [aOR] 4.93; 95\% confidence interval [Cl]:1.56 to 15.63 vs. $2.79 ; 95 \% \mathrm{Cl}, 1.12$ to 6.97 , respectively), and only the ICDSC significantly predicted hospital LOS with a mean of 17.59 additional days compared to the no-delirium group. Irrespective of delirium status, a sensitivity analysis of normal-to-increased arousal (RASS $\geq 0$ ) test results did not alter the predictive ability of ICDSC- or CAM-ICU-delirium for hospital mortality (aOR 2.97; $95 \% \mathrm{Cl}, 1.06$ to 8.37 vs. 3.82; $95 \% \mathrm{Cl}, 1.35$ to 10.82 , respectively). With reduced arousal (RASS $<0)$, neither tool significantly predicted mortality or LOS.

Conclusions: The ICDSC identified more delirium cases and may have higher predictive validity for mortality and LOS than the CAM-ICU. However, arousal substantially affected performance. Future studies may want to consider patients' arousal when deciding which tool to use to maximize the effects of delirium identification on patient mortality.

Trial registration: NCT 04206306

\section{Introduction}

Intensive care unit (ICU)-acquired delirium impacts patient outcomes, i.e., higher mortality [1-6] and prolonged length hospital of stay (LOS) [1-3, 6-9]. Delirium can be accurately identified by widely validated screening tools such as the intensive care delirium-screening checklist (ICDSC) and the confusion assessment method for the ICU (CAM-ICU) [10,11], but delirium in ICU settings is still poorly recognized [12]. Only $40 \%$ of clinicians consistently screen for ICU delirium [13], and clinically effective screening tools are not consistently applied [14]. 
To encourage consistent application of effective ICU-acquired delirium screening tools, we considered that a head-to-head comparison of tools might help to guide tool selection. While both the ICDSC and CAM-ICU have been recommended by the Society of Critical Care Medicine for detecting ICU delirium [15], it remains unclear which tool is more clinically relevant. Their ability to predict delirium-related outcomes such as mortality and LOS warrants investigation.

Outcomes in ICU settings are affected by many factors. Mortality may be more strongly influenced by altered arousal (either reduced or increased) than by delirium diagnosis alone [16]. Indeed, a six-study meta-analysis found that altered arousal in patients with delirium was associated with higher mortality than normal arousal in patients with or without delirium [16]. Moreover, reduced arousal may interfere with delirium diagnosis, especially when using screening tools such as the ICDSC and the CAM-ICU. For example, reduced arousal has been suggested to overidentify delirium [17], as delirium rates were reduced significantly if test results from patients on the Richmond Agitation-Sedation Scale (RASS) scores -2 to -3 were excluded [18]. The authors of that study noticed that a lower RASS score ( 2 or deeper) tended to fulfill the delirium diagnosis, and delirium rates increased by approximately a quarter to a third [18]. In short, when studying the impact of delirium on mortality, reduced arousal, irrespective of delirium status, is an important confounder requiring further study.

In this prospective cohort study, we aimed to compare the predictive validity of the ICDSC versus CAM-ICU in predicting delirium-related outcomes of hospital mortality and LOS. The time spent being tested with each tool, as a proxy for their clinical utility, was also compared. Moreover, we examined whether the tools' predictive validity and time spent in tests were affected by patients' arousal status.

\section{Materials And Methods}

After this prospective cohort study was approved by the Institutional Review Board and registered in the clinical trial registry (NCT 04206306), we enrolled consecutive adult patients ( $\geq 20$ years) who stayed > 24 hours in six medical ICUs at a university affiliated hospital in Taiwan from December 2019 to October 2020. Patients were excluded by these criteria: 1) had pre-existing delirium;2) were bedridden, had moderate dementia (clinical dementia rating [CDR] score $\geq 2$ ), had severe hearing impairment, or could not communicate; or 3) were placed on contact and droplet precaution. All patients or their surrogates signed written informed consent forms to participate in the study. The detailed study flow chart is in Fig. 1.

\section{Data Collection}

A standardized data entry form was developed to collect all participants' clinical data: age (years), sex (\%), education level (\%; under high school; high school and above), very mild or mild dementia (\%; defined by $\mathrm{CDR}=0.5$ or 1 ), Charlson comorbidity index $(\mathrm{CCl})$ score, ICU admission diagnosis (acute respiratory failure; noncardiogenic shock; cardiac emergency; other), use of mechanical ventilation (MV; yes/no), and the acute physiology and chronic health evaluation (APACHE II) score (24 hours within admission). 
Regarding study outcomes, in-hospital mortality and LOS in the hospital were abstracted from the medical record.

\section{Delirium Assessment}

Research nurses screened patients daily and up to 14 days of ICU stay sequentially with the ICDSC and CAM-ICU; the nurses' screening for ICU delirium was calibrated with an experienced psychiatrist. To limit bias introduced by the same assessor's consecutive evaluation with two similar instruments, a random order was created using the REDCap data collection system and strictly followed to determine which tool was administered first at each paired assessment.

Before each delirium screening, participants' arousal was evaluated by the RASS, with scores ranging between -5 (unarousable) and $\mathbb{8} 4$ (combative). For arousable participants (RASS -3 and higher), delirium was assessed by both the ICDSC and CAM-ICU. Briefly, based on Diagnostic and Statistical Manual of Mental Disorders (DSM-IV) criteria, the ICDSC is an 8-item checklist of delirium symptoms (consciousness, inattention, disorientation, hallucinations/delusions/psychosis, psychomotor agitation or retardation, inappropriate speech or mood, sleep/wake cycle disturbances, and symptom fluctuation); any 4 items presented within a 24-hour time frame indicate delirium [19]. The CAM-ICU consists of 4 consecutive items (1-acute change/fluctuation in mental status, 2-inattention, 3-altered level of consciousness, 4-disorganized thinking); at least 3items $(1+2+3$ or $1+2+4)$ must be presented to indicate delirium [20]. In this study, participants with any one positive result during the first 14 days of their ICU stay were classified accordingly into ICDSC- or CAM-ICU-delirium groups.

\section{Outcomes: Mortality, Los, And Time Spent Administering Tools}

The primary outcomes were in-hospital mortality and hospital LOS, abstracted from medical records. The secondary outcome, time spent (minutes) administering the ICDSC versus the CAM-ICU was automatically recorded for each paired test administered with a built-in random tool order. Notably, for both tools, we did not count the time trained research nurses spent to collect participant data in the last 24 hours from the chart or from the primary nurse, given that the same assessor consecutively evaluated these two tools; only time spent in rating tool items was recorded for comparison.

\section{Statistical analysis}

Data were analyzed using SPSS 22.0 (IBM, New York, NY), with all tests being two-tailed and $p<0.05$ considered significant. The sample was described by means and standard deviations (SDs) for continuous variables, with counts and percentages for categorical variables. The degree of agreement between the ICDSC and CAM-ICU was measured using kappa (K). Agreement was defined as moderate 
(0.41-0.60), substantial (0.61-0.80), or perfect (>0.80) [21]. Mortality risk and LOS in the ICDSC- $v s$. CAM-ICU-delirium cohorts were estimated using multiple logistic and linear regressions models.

All models were adjusted for relevant confounders, including age, APACHE II, and CCI scores, to obtain adjusted odds ratios (aORs) and adjusted $\beta$. To test whether arousal state changed the predictive ability of delirium for mortality and LOS, we conducted sensitivity analyses to stratify normal-to-increased arousal (RASS $\geq 0$ ) versus reduced arousal (RASS $<0$ ) test results, irrespective of delirium status. In fact, a RASS score of $\geq 0$ indicates either altered or aggressive symptomatology; thus, we defined RASS $\geq 0$ as "normal-to-increased arousal," with negative RASS scores of -1 to -3 indicating drowsiness and being defined as "reduced arousal."

\section{Results}

Of 384 adult patients admitted to medical ICUs and prospectively screened, 97 participants were included in the analysis. Details of the study flow chart are in Fig. 1. As shown in Table 1, 97 participants had a mean (SD) age of 67.1 (13.9) years, $67 \%$ were male, and $55.7 \%$ (54/97) had a high school education level or above. Only $3(3.1 \%)$ had very mild or mild dementia (CDR score $=0.5$ or 1 ). The participants' comorbidity burden was high (mean (SD) CCI score $=3.9(2.3)$ points). The most common ICU admission diagnosis was acute respiratory failure $(51 / 97 ; 52.6 \%)$, followed by noncardiogenic shock $(35 / 97 ; 36.1 \%)$, cardiac emergency $(7 / 97 ; 7.2 \%)$, and other $(4 / 97 ; 4.2 \%)$. The mean (SD) APACHE II score at 24 hours after ICU admission was 22.7 (7.2), and 66/97 (68\%) of patients received mechanical ventilation (MV).

Ultimately, 35 patients died, resulting in an in-hospital mortality rate of $36.5 \%$ (35/96). The mean (SD) LOS was 40.6 (32.2) days. Notably, in 571 daily paired delirium screenings over a mean (SD) of 5.9 (4.0) days, the time spent screening with the ICDSC and CAM-ICU did not differ significantly (mean minutes [SD], 1.1 [0.9] vs. 1.1 [0.8] for CAM-ICU; $p=0.96$ ). 
Table 1

Sample characteristics and outcomes

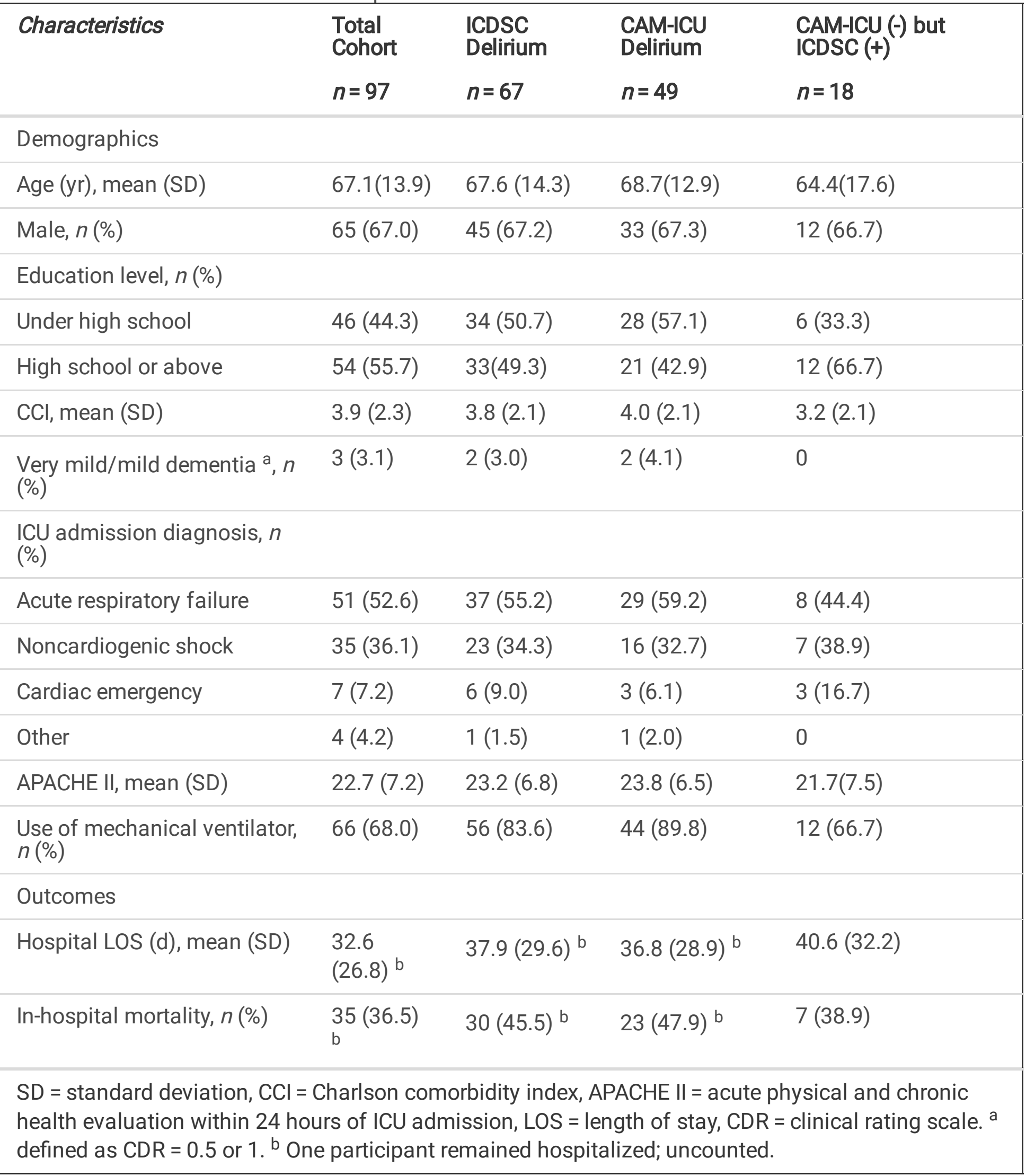

ICDSC vs. CAM-ICU: concordance and predictive validity 
As shown in Table 2,67/97 participants were classified with ICDSC delirium, resulting in a delirium incidence of $69.1 \%$. Conversely, only 49 participants were classified with CAM-ICU delirium, resulting in a delirium incidence of $50.5 \%$. Although the ICDSC identified 18 more delirium cases than the CAM-ICU, concordance ( $\mathrm{K}=0.63 ; p<0.001)$ was substantial between the ICDSC and CAM-ICU for detecting delirium given their complete agreement on 49 delirium cases. A detailed $2 \times 2$ contingency table is provided in Supplemental Table $1 \mathrm{a}$.

Table 2

Incidence and kappa between ICDSC- and CAM-ICU-identified delirium cases

\begin{tabular}{|c|c|c|c|}
\hline & $\begin{array}{l}\text { ICDSC Delirium, } n \\
(\%)\end{array}$ & $\begin{array}{l}\text { CAM-ICU Delirium, } n \\
(\%)\end{array}$ & $\begin{array}{l}\text { Cohen's kappa } \\
\text { (K) }{ }^{a}\end{array}$ \\
\hline All participants & $67 / 97(69.1)$ & $49 / 97(50.5)$ & 0.63 \\
\hline $\begin{array}{l}\text { Stratified by normal-to-increased } \\
\text { arousal }\end{array}$ & $33 / 87$ (37.9) & 27/87 (31.0) & 0.75 \\
\hline Stratified by reduced arousal & $59 / 74(79.7)$ & $42 / 74(56.8)$ & 0.44 \\
\hline
\end{tabular}

Furthermore, as shown in Table 3, the ICDSC-delirium cohort had higher in-hospital mortality (45.5\% vs. $16.7 \%$ for the no ICDSC-delirium group; $p=0.007$ ) and longer hospital LOS (mean days [SD], 37.9 [29.6] vs. 20.9 [13.47] for the no ICDSC-delirium group; $p=0.004$ ). In the CAM-ICU group, participants had higher in-hospital mortality $(47.9 \%$ vs. $25.0 \%$ for the no-delirium group; $p=0.02)$ but a comparable LOS (mean days [SDs], 36.8 [28.9] vs. 28.3 [24.1] for the no delirium group; $p=0.12$ ). Logistic regression models revealed that belonging to the ICDSC-delirium group was associated with a 4.93-fold (aOR) higher inhospital mortality $(95 \% \mathrm{Cl}, 1.56-15.63 ; p=0.007)$ than the 2.79 -fold mortality risk for those in the CAMICU delirium group $(95 \% \mathrm{Cl}, 1.12-6.97 ; p=0.028)$. For LOS, linear regression analyses indicated that belonging to the ICDSC-delirium group was associated with prolonged LOS; participants in the ICDSCdelirium group stayed 17.59 days longer in the hospital (adjusted $\beta$ [95\% Cl], 17.59 [6.11-29.09]; $p=$ 0.003 ). For the CAM-ICU delirium group, LOS was longer but not significantly so (adjusted $\beta[95 \% \mathrm{CI}], 8.49$ [-2.61-19.59]; $p=0.13)$. 
Table 3

All participants: ICDSC-Delirium and CAM-ICU-Delirium on hospital mortality and LOS

\begin{tabular}{|c|c|c|c|c|c|c|c|c|}
\hline \multirow{2}{*}{$\begin{array}{l}\text { In-Hospital } \\
\text { Mortality }\end{array}$} & \multicolumn{3}{|c|}{ Delirium } & \multicolumn{3}{|c|}{ No Delirium } & \multirow{2}{*}{$\begin{array}{l}\text { Adjusted } \\
\text { odds ratio }^{b}\end{array}$} & \multirow[t]{2}{*}{$95 \% \mathrm{Cl}$} \\
\hline & Total & Death & $(\%)$ & Total & Death & $(\%)$ & & \\
\hline ICDSC & 67 & 30 & $\begin{array}{l}(45.5) \\
a\end{array}$ & 30 & 5 & $(16.7)$ & 4.93 & $\begin{array}{l}(1.56- \\
15.63) \\
\star\end{array}$ \\
\hline CAM-ICU & 49 & 23 & $\begin{array}{l}(47.9) \\
a\end{array}$ & 48 & 12 & $(25.0)$ & 2.79 & $\begin{array}{l}(1.12- \\
6.97) \text { * }\end{array}$ \\
\hline \multirow[t]{2}{*}{ LOS } & \multicolumn{3}{|c|}{ Delirium } & \multicolumn{3}{|c|}{ No Delirium } & \multirow{2}{*}{$\begin{array}{l}\text { Adjusted } \\
\text { difference }^{b}\end{array}$} & \multirow[t]{2}{*}{$95 \% \mathrm{C}$} \\
\hline & $\begin{array}{l}\text { Mean } \\
a\end{array}$ & $(S D)^{a}$ & & Mean & $(S D)$ & & & \\
\hline ICDSC & $\begin{array}{l}37.9 \\
a\end{array}$ & $(29.6)$ & & 20.9 & $(13.7)$ & & 17.59 & $\begin{array}{l}(6.11- \\
29.09)\end{array}$ \\
\hline CAM-ICU & $\begin{array}{l}36.8 \\
a\end{array}$ & $(28.9)$ & & 28.3 & $(24.1)$ & & 8.49 & $\begin{array}{l}(-2.61- \\
19.59)\end{array}$ \\
\hline \multicolumn{9}{|l|}{$* p<0.05$} \\
\hline \multicolumn{9}{|c|}{ a One participant remained hospitalized; uncounted. } \\
\hline
\end{tabular}

\section{Sensitivity Analysis - Effect Of Arousal}

As arousal may impact not only delirium diagnosis but also its predictive validity, we stratified patients by normal-to-increased arousal (RASS $\geq 0$ ) vs. reduced arousal (RASS $<0$ ), irrespective of delirium status, to determine whether the findings were consistent. Delirium rates varied between arousal groups (Table 2). For both tools, delirium incidence was lower in the RASS $\geq 0$ subgroup ( 272 daily paired screenings), whereas it was higher in the RASS $<0$ subgroup (299 daily paired screenings). This trend was evident, especially for the ICDSC-delirium group (79.7\% incidence for the reduced arousal subgroup vs. $37.9 \%$ for the normal-to-increased arousal subgroup). Similarly, concordance also varied between arousal groups. A much higher concordance $(\mathrm{K}=0.75 ; p<0.001)$ was reported for RASS $\geq 0$, while a lower concordance $(\mathrm{K}=$ 0.44 ) was reported for RASS $<0$.

The prognostic difference in mortality between arousal states also varied (Table 4). Both tools' predictive validity for mortality diminished when stratified by reduced arousal (RASS $<0)$. For normal-to-increased arousal, the CAM-ICU-delirium group had higher mortality odds than the ICDSC-delirium group (aOR, 3.82 vs. 2.97 for ICDSC), although both tools predicted hospital mortality. For LOS, neither the ICDSC nor CAM- 
ICU predicted this outcome, regardless of arousal state. Similarly, for each tool, the time spent screening for delirium did not differ. As expected, the mean (SD) time spent screening for delirium in the RASS $<0$ subgroup was longer than in the RASS $\geq 0$ subgroup for both tools: $1.3(0.9) v s .1 .0(0.9)$ min for the ICDSC and $1.2(0.8)$ vs. $1.0(0.8)$ min for the CAM-ICU. 
Table 4

Stratify by arousal: ICDSC-Delirium and CAM-ICU-Delirium on hospital mortality and LOS Normal-to-Increased arousal (RASS $\geq 0$ subgroup)

\begin{tabular}{|c|c|c|c|c|c|c|c|c|}
\hline \multirow{2}{*}{$\begin{array}{l}\text { In-hospital } \\
\text { mortality }\end{array}$} & \multicolumn{3}{|c|}{ Delirium } & \multicolumn{3}{|c|}{ No Delirium } & \multirow{2}{*}{$\begin{array}{l}\text { Adjusted } \\
\text { odds ratio } \\
\text { b }\end{array}$} & \multirow[t]{2}{*}{$95 \% \mathrm{Cl}$} \\
\hline & Total & Death & $(\%)$ & Total & Death & $(\%)$ & & \\
\hline ICDSC & 33 & 15 & $\begin{array}{l}(46.9) \\
\mathrm{a}\end{array}$ & 54 & 13 & $(24.1)$ & 2.97 & $\begin{array}{l}(1.06- \\
8.37)^{*}\end{array}$ \\
\hline CAM-ICU & 27 & 14 & $\begin{array}{l}(53.8) \\
\mathrm{a}\end{array}$ & 60 & 14 & (23.3) & 3.82 & $\begin{array}{l}(1.35- \\
10.82)\end{array}$ \\
\hline \multirow[t]{2}{*}{ LOS } & \multicolumn{3}{|c|}{ Delirium } & \multicolumn{3}{|c|}{ No Delirium } & Adjusted & $95 \% \mathrm{Cl}$ \\
\hline & $\begin{array}{l}\text { Mean } \\
\mathrm{a}\end{array}$ & $(S D)^{a}$ & & Mean & (SD) & & b & \\
\hline ICDSC & $\begin{array}{l}35.5 \\
a\end{array}$ & (29.9) & & 30.5 & $(25.4)$ & & 5.12 & $\begin{array}{l}(-7.84- \\
18.07)\end{array}$ \\
\hline CAM-ICU & $\begin{array}{l}32.3 \\
a\end{array}$ & $(24.6)$ & & 32.4 & (28.3) & & -1.12 & $\begin{array}{l}(-14.38- \\
12.13)\end{array}$ \\
\hline
\end{tabular}

Reduced arousal (RASS $<0$ subgroup)

\begin{tabular}{|c|c|c|c|c|c|c|c|c|}
\hline \multirow{2}{*}{$\begin{array}{l}\text { In-hospital } \\
\text { mortality }\end{array}$} & \multicolumn{3}{|c|}{ Delirium } & \multicolumn{3}{|c|}{ No Delirium } & \multirow{2}{*}{$\begin{array}{l}\text { Adjusted } \\
\text { odds ratio } \\
\text { b }\end{array}$} & \multirow[t]{2}{*}{$95 \% \mathrm{Cl}$} \\
\hline & Total & Death & $(\%)$ & Total & Death & $(\%)$ & & \\
\hline ICDSC & 59 & 27 & $\begin{array}{l}(46.6) \\
a\end{array}$ & 15 & 5 & (33.3) & 1.65 & $\begin{array}{l}(0.47- \\
5.86)\end{array}$ \\
\hline CAM-ICU & 42 & 20 & $\begin{array}{l}(48.8) \\
a\end{array}$ & 32 & 12 & (37.5) & 1.33 & $\begin{array}{l}(0.48- \\
3.66)\end{array}$ \\
\hline \multirow[t]{2}{*}{ LOS } & \multicolumn{3}{|c|}{ Delirium } & \multicolumn{3}{|c|}{ No Delirium } & Adjusted & $95 \% \mathrm{Cl}$ \\
\hline & $\begin{array}{l}\text { Mean } \\
a\end{array}$ & $(S D)^{a}$ & & Mean & (SD) & & & \\
\hline ICDSC & $\begin{array}{l}38.3 \\
a\end{array}$ & $(30.8)$ & & 25.5 & $(18.6)$ & & 13.19 & $\begin{array}{l}(-3.74- \\
30.13)\end{array}$ \\
\hline
\end{tabular}

${ }^{\star} p<0.05$

a One participant remained hospitalized; uncounted.

b All models were adjusted for age, APACHE II, and CCI scores. 


\section{Normal-to-Increased arousal (RASS $\geq 0$ subgroup)}
CAM-ICU
38.2
$(30.2)$
32.6
(27.8)
4.72
$(-9.40-$
a
$18.84)$

${ }^{*} p<0.05$

a One participant remained hospitalized; uncounted.

b All models were adjusted for age, APACHE II, and CCI scores.

\section{Discussion}

The most important finding of our study is that ICU delirium identified by either screening tool, the ICDSC or CAM-ICU, was linked with increased deaths during hospitalization. The ICDSC outperformed the CAMICU because it identified delirium with higher odds of mortality and predicted hospital LOS. However, the performance of both tools was affected by patient arousal.

Two findings are worth emphasizing. First, as delirium incidence rates evaluated by the ICDSC and CAMICU were $69.1 \%$ and $50.5 \%$, respectively, and the ICDSC identified 18 more cases of delirium, we found that a wider net was cast by the ICDSC, capturing more delirium cases than the CAM-ICU. Our result is consistent with that of the only study available for comparison, a Brazilian study of 162 surgical ICU patients whose delirium rate evaluated by the ICDSC was $34.5 \%(n=56)$ vs. $26.5 \%(n=43)$ by the CAMICU [22]. This wider net cast by the ICDSC may be welcome by certain institutions but may also bring additional diagnostic and care burden and fatigue to the nursing and physician staff as more cases were identified.

We thus focused on the tools' relative predictive validity for important outcomes and found that the ICDSC identified delirium had higher predictive validity for both mortality and LOS than the CAM-ICU. Participants in the ICDSC-delirium group had a 4.93-fold higher mortality risk and stayed at the hospital 17.59 days longer than those in the no-delirium group, while those in the CAM-ICU group were linked with a 2.79-fold lower odds of mortality. This finding is consistent with prior reports that ICU delirium increased hospital mortality in patients evaluated by either the ICDSC [23-25] or CAM-ICU [5, 6, 27-28], but different from a previous finding that the CAM-ICU better predicted outcome [22]. The reason for this difference requires further study, but one factor to consider is participants' arousal state.

This leads to our second point. For both the ICDSC and CAM-ICU, reduced arousal affects performance. Although both the ICDSC and CAM-ICU can be used when patients' RASS level was -1 to -3 (awakening to voice), their performance seemed to be less stable. Namely, agreement between tools is low ( $\mathrm{K}=0.44$ at RASS -1 to -3 compared to 0.75 at RASS $\geq 0$ ). Moreover, if used with patients in reduced arousal states $(-3 \leq$ RASS $<0)$, both the ICDSC and CAM-ICU tended to identify delirium cases at a higher rate (Table 2) than with patients in normal-to-increased arousal. This trend is particularly apparent for the ICDSC, as its delirium incidence reached $79.7 \%$, representing a $15 \%$ increased incidence from the sample mean. With 
this higher rate of delirium, neither ICDSC- nor CAM-ICU-identified delirium predicted hospital mortality or LOS. This loss of predictive validity is noteworthy.

Why delirium identified in the reduced arousal subgroup was not associated with mortality and LOS is an important research question. The diminished effect on mortality may be due to misclassification of delirium cases (e.g., more false positives), resulting in reduced analytic power. Moreover, when participants have RASS levels between -1 and -3 , the ICDSC and CAM-ICU may measure pure sedation effects. As decreased arousal is likely be multifactorial, combining both non-serious and serious conditions (e.g., medication/sedation effects versus serious neurologic events) may thus exert less consistent prognostic effects on mortality and/or LOS. Lastly, our study may be underpowered to detect the desired difference due to a relatively small sample. Future studies with larger sample sizes are indicated to verify our results.

Apart from the over-identification perspective, a wider spectrum has been recommended in delirium diagnosis (i.e., more inclusive recognition of delirium). For example, a 14-study meta-analysis $(21,198$ medical admission patients) found that reduced arousal (mostly defined by the Glasgow Coma Scale; only one with RASS) on hospital admission was associated with 5.7-fold greater mortality rates [29]. In that study, the authors argued that as delirium and reduced arousal are closely related and both are linked with high mortality, delirium studies should include patients who are too drowsy to undergo cognitive testing or interviews. Otherwise, the restricted spectrum (by eliminating patients with reduced arousal) may have led to underestimating the relationship between delirium and mortality [29].

Nevertheless, consistent with prior studies, ICU delirium evaluated by the ICDSC and CAM-ICU demonstrated substantial diagnostic agreement, and both tools could be completed in a comparable time, slightly over 1 minute in our study. A more sensitive screening tool, such as the ICDSC, holds promise by casting a wider net and capturing more delirium cases than the CAM-ICU. Whether systematically using the ICDSC changes predicted outcomes requires an impact-evaluation study. Moreover, given that the two tools' agreement and predictive validity were much lower in the reduced arousal subgroup, future studies with larger samples may want to account for patients' arousal when deciding which tool to use to maximize the effects of delirium identification on patient mortality.

\section{Study Strength And Limitations}

Our findings should be considered with certain limitations, including potential confounders and misclassification biases. As participants with any one positive result were classified into the delirium group, "days being assessed" was a confounder inherent in our study design; participants who stayed in the ICU longer were more likely to be classified into the delirium group. Moreover, the impact of delirium was assumed to be equal irrespective of its duration. Second, the same rater completed both measures so their administration could not be blinded to each other. The randomization of ordering was important but cannot overcome this limitation, which should be acknowledged. Third, delirium has no gold standard for diagnosis; thus, the "truth" of which measure better reflected delirium cannot be definitely established. 
Along these lines, we acknowledge the limitation that delirium was screened by well-trained research nurses instead of an experienced psychiatrist. However, both the ICDSC and CAM-ICU have been designed and used by nonpsychiatric personnel, especially nurses, with high reliability. Fourth, our study was limited by its small sample and power may have been limited for many of the analyses. Moreover, the study was limited by enrolling participants from a single institution with an enrolment rate of $83.3 \%$ (100 of 120 invited eligible patients). While all eligible patients were offered enrollment, selection bias remains a limitation that will need to be addressed by replicating the findings in future studies.

\section{Conclusions}

Delirium, identified either by the ICDSC or CAM-ICU, was linked with hospital death in critically ill patients. A head-to-head comparison indicated that the ICDSC identified delirium with higher predictive validity for both mortality and LOS than the CAM-ICU. However, this evaluation may change based on level of arousal. More studies are needed to consider ICU patients' arousal when deciding which tool truly maximizes the effects of delirium identification on patient mortality.

\section{Abbreviations}

ICDSC

Intensive care delirium screening checklist; CAM-ICU:Confusion assessment method for intensive care unit; RASS:Richmond Agitation-Sedation scale; LOS:length of stay; VS:Versus; CCI:Charlson comorbidity index; APACHE II:Acute Physical and Chronic Health Evaluation; CDR:Clinical rating scale.

\section{Declarations}

Acknowledgements: The authors would like to acknowledge the help provided by Dr. Yu-Juan Xu and Mr. Chi-Hsuan Su in the study recruitment and data collection.

\section{Authors' contributions}

HL screened participants' delirium, analyzed the data, and draft the manuscript.

CC conceived of the study, participated in data collection, and helped to draft the manuscript.

TY participated in study design, funding and coordination.

SL participated in study design and coordination

AH cross-validated participants' delirium

YW performed the statistical analysis

SS participated in study design

SK conceived of the study and supervised the data collection in all clinical sites. 
SI participated in study design, interpreted the data, and helped to draft the manuscript.

All authors read and approved the final manuscript.

Funding: This study was supported in part by grants 107-2314-B-002-023-MY3 from the Taiwan Ministry of Science and Technology. Dr. Inouye's time was supported in part by grant no. R33AG071744 from the U.S. National Institute on Aging; Dr. Inouye also holds the Milton and Shirley F. Levy Family Chair at Hebrew Senior Life/Harvard Medical School.

Availability of data and materials: All data generated and/or analyzed during the current study are included within the published article and its additional files.

\section{Ethics approval and consent to participate}

The prospective cohort study was approved by the Institutional Review Board and registered in the clinical trial registry (NCT 04206306). All patients or their surrogates signed written informed consent forms to participate in the study.

Consent for publication: Not applicable.

Competing interests: The authors declare that they have no competing interests.

\section{References}

1. Aung Thein MZ, Pereira JV, Nitchingham A, Caplan GA. A call to action for delirium research: metaanalysis and regression and regression of delirium association mortality. BMC Geriatr. 2020;20:32535.

2. Salluh JIF, Wang H, Schneider EB, Nagaraja N, Yenokyan G, Damluji A, et al.Outcome of delirium in critically ill patients: systematic review and meta-analysis. BMJ. 2015; 350.

3. Abelha FJ, Luís C, Veiga D, Fernandes V, Santos P, Botelho M, et al. Outcome and quality of life in patients with postoperative delirium during an ICU stay following major surgery. Crit Care. 2013;17:R257.

4. Ali MA, Hashmi M, Ahmed W, RazaSA, Khan MF, Salim B. Incidence and risk factors of delirium in surgical intensive care unit. J Trauma Acute Care Surg. 2021;6:e000564.

5. Pauley E, Lishmanov A, Schumann S, Gala GJ, Diepen S, Katz JN. Delirium is a robust predictor of morbidity and mortality among critically ill patients treated in the cardiac intensive care unit. Am Heart J. 2015;170:79-86.

6. Zhang R, Bai L, Han X, Huang S, Zhou L, Duan J. Incidence, characteristics, and outcomes of delirium in patients with noninvasive ventilation: a prospective observational study. BMC Pulm Med. 2021;21:157-64. 
7. Dziegielewski C, Skead C, Canturk T, Webber C, Fernando SM, Thompson LH, et al. Delirium and associated length of stay and costs in critically. Crit Care Res Pract. 2021. https://doi.org/10.1155/2021/6612187.

8. Boettger S, Meyer R, Richter A, Fernandez SF, Rudiger A, Schubert M, et al. Screening for delirium with the intensive care delirium screening checklist (ICDSC): symptom profile and utility of individual items in the identification of delirium dependent on the level of sedation. Palliat Support Care. 2019;17:74-81.

9. Kirfel A, Menzenbach J, Guttenthaler V, Feggeler J, Mayr A, Coburn M, Wittmann M. Postoperative delirium after cardiac surgery of elderly patients as an independent risk factor for prolonged length of stay in intensive care unit and in hospital. Aging Clin Exp Res. 2021. https://doi.org/10.1007/s40520-021-01842-x.

10. Gusmao-Flores D, Salluh JIF, Chalhub R, Quarantini LC. The confusion assessment method for the intensive care unit (CAM-ICU) and intensive care delirium screening checklist (ICDSC) for the diagnosis of delirium: a systematic review and meta-analysis of clinical studies. Crit Care. 2012;16:R115.

11. Chen TJ, Chung YW, Chang HC, Chene PY, Wua CR, Hsieha SH, et al. Diagnostic accuracy of the CAMICU and ICDSC in detecting intensive care unit delirium: A bivariate meta-analysis. Int J Nurs Stud. 2021;113:103782.

12. Spronk PE, Riekerk B, Hofhuis J, Rommes JH. Occurrence of delirium is severely underestimated in the ICU during daily care. Intensive Care Med. 2009;35:1276-80.

13. Ely EW, Stephens RK, Jackson JC, Jackson JC, Thomason JWW, Truman B, et al. Current opinions regarding the importance, diagnosis, and management of delirium in the intensive care unit: a survey of 912 healthcare professional. Crit Care Med. 2004;32:106-12.

14. Khachaturian AS, Hayden KM, Devlin JW, Fleisher LA, Lock SL, Cunningham C, et al. International drive to illuminate delirium: A developing public health blueprint for action. J Alzheimers Dis. 2020;16:711-25.

15. Barr J, Pandharipande PP. The pain, agitation, and delirium care bundle: synergistic benefits of implementing the 2013 pain, agitation, and delirium guidelines in an integrated and interdisciplinary fashion. Crit Care Med. 2013;41:99-115.

16. Tieges Z, Quinn T, Mackenzie L, Davis D, Muniz-Terrera G, MacLullich AMJ, et al. Association between components of the delirium syndrome and outcome in hospitalised adults: a systematic review and meta-analysis. BMC Geriatr. 2021;21:162-75.

17. Gusmao-Flores D, Martins JCS, Amorin D, Quarantini LC. Tools for diagnosing delirium in the critically ill: is calibration needed for the less sedated patient? Intensive Care Med. 2014;40:137-8.

18. Haenggi M, Blum S, Brechbueh R, Brunello A, Jakob SM, Takala J. Effect of sedation level on the prevalence of delirium when assessed with CAM-ICU and ICDSC. Intensive Care Med. 2013;39:21719. 
19. Bergeron N, Dubois MJ, Dumont M, Dial S, Skrobic Y. Intensive Care Delirium Screening Checklist: evaluation of a new screening tool. Intensive Care Med. 2001;27:859-64.

20. Ely EW, Inouye SK, Bernard GR, Gordan S, Francis J, May L, et al. Delirium in mechanically ventilated patients: validity and reliability of the confusion assessment method for the intensive care unit (CAM-ICU). JAMA. 2001;286:2703-10.

21. Landis JR, Koch GG. The measurement of observer agreement for categorical data. Biometrics. 1977;159-174.

22. Tomasi CD, Grandi C, Salluh J, Soraes M, Giombellia VR, Cascaesa R, et al. Comparison of CAM-ICU and ICDSC for the detection of delirium in critically ill patients focusing on relevant clinical outcomes. J Crit Care. 2012;27:212-7.

23. Dittrich T, Tschudin-Sutter S, Widmer AF, Rüegg S, Marsch S, Sutter R. Risk factors for new-onset delirium in patients with bloodstream infections: independent and quantitative effect of catheters and drainages-a four-year cohort study. Ann Intensive Care. 2017;6:104.

24. Ouimet S, Kavanagh BP, Gottfried SB, Skrobik Y. Incidence, risk factors and consequences of ICU delirium. Intensive Care Med. 2007;33:66-73.

25. Yamaguchi T, Tsukioka E, Kishi Y. Outcomes after delirium in a Japanese intensive care unit. Gen Hosp Psychiatry. 2014;36:634-6.

26. Kim Y, Jin Y, Jin T, Lee SM. Risk factors and outcomes of sepsis-associated delirium in intensive care unit patients: a secondary data analysis. Intensive Crit Care Nurs. 2020;59:102844.

27. van den Boogaard M, Peters SA, van der Hoeven JG, Dagnelie PC, Leffers P, Pickkers P, et al. The impact of delirium on the prediction of in-hospital mortality in intensive care patients. Crit Care. 2010;14:R146.

28. Lin SM, Huang CD, Liu CY, Lin HC, Wang CH, Huang PY,et al. Risk factors for the development of early-onset delirium and the subsequent clinical outcome in mechanically ventilated patients. $\mathrm{J}$ Crit Care. 2008;23:372-9.

29. Todd A, Blackley S, Burton JK, Stott DJ, Ely EW, Tieges Z, et al. Reduced level of arousal and increased mortality in adult acute medical admissions: a systematic review and meta-analysis. BMC Geriatr. 2017;17:283.

\section{Figures}




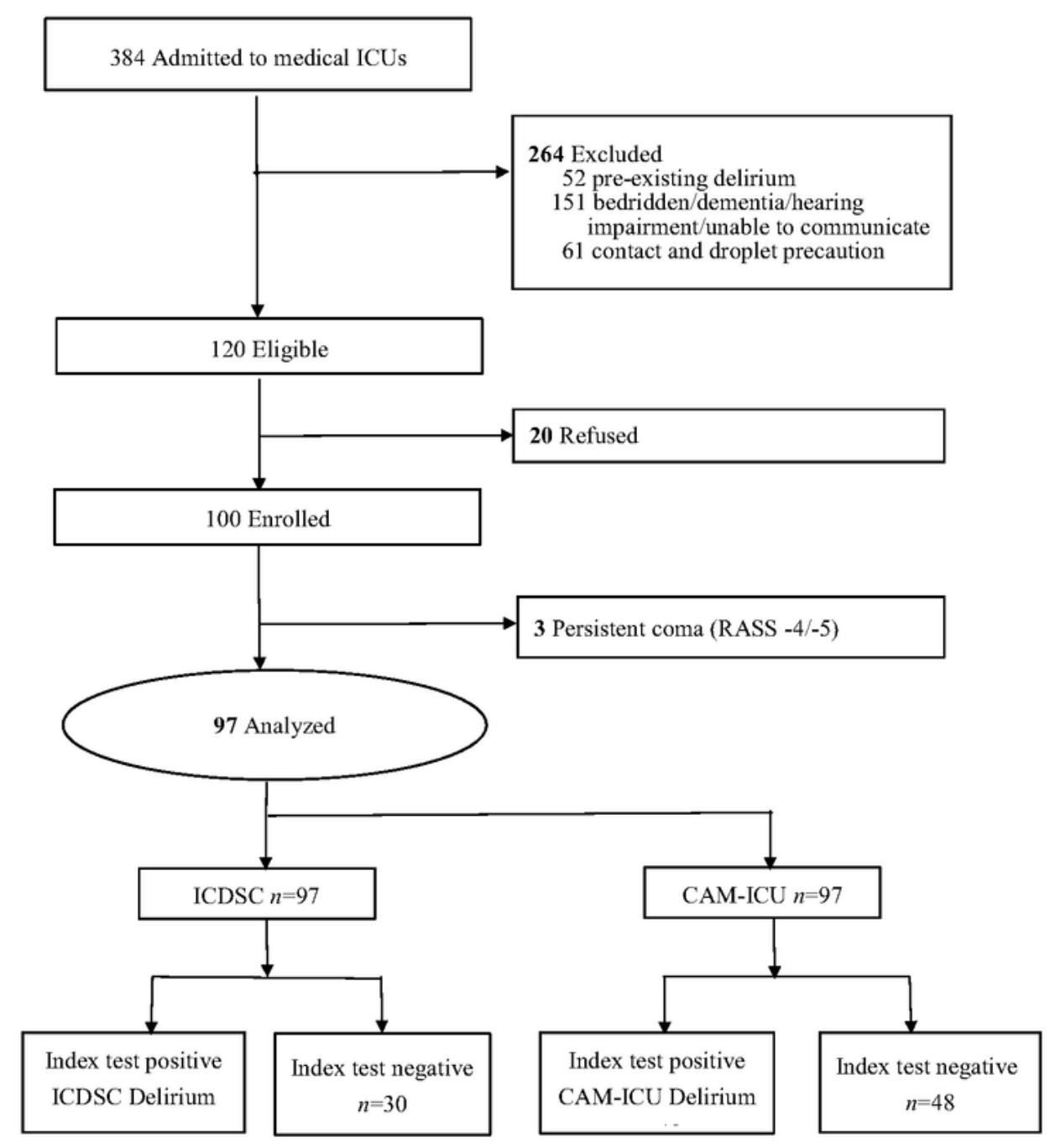

Figure 1

Study flowchart.

\section{Supplementary Files}

This is a list of supplementary files associated with this preprint. Click to download. 
- 8SupplementalTable.docx

Page 18/18 